\title{
Neutron reflectivity measurements at the oil/water interface for the study of stimuli-responsive emulsions
}

DOI:

10.1140/epje/i2018-11693-8

\section{Document Version}

Accepted author manuscript

Link to publication record in Manchester Research Explorer

\section{Citation for published version (APA):}

Protat, M., Bodin-Thomazo, N., Malloggi, F., Daillant, J., Campbell, R. A., Fragneto, G., Watkins, E. B., Perrin, P., Pantoustier, N., \& Guenoun, P. (2018). Neutron reflectivity measurements at the oil/water interface for the study of stimuli-responsive emulsions. European Physical Journal E, 41(85). https://doi.org/10.1140/epje/i2018-11693-8

\section{Published in:}

European Physical Journal E

\section{Citing this paper}

Please note that where the full-text provided on Manchester Research Explorer is the Author Accepted Manuscript or Proof version this may differ from the final Published version. If citing, it is advised that you check and use the publisher's definitive version.

\section{General rights}

Copyright and moral rights for the publications made accessible in the Research Explorer are retained by the authors and/or other copyright owners and it is a condition of accessing publications that users recognise and abide by the legal requirements associated with these rights.

\section{Takedown policy}

If you believe that this document breaches copyright please refer to the University of Manchester's Takedown Procedures [http://man.ac.uk/04Y6Bo] or contact uml.scholarlycommunications@manchester.ac.uk providing relevant details, so we can investigate your claim.

\section{OPEN ACCESS}




\title{
Neutron reflectivity measurements at the oil/water interface for the study of stimuli-responsive emulsions
}

\author{
M. Protat ${ }^{1}$, N. Bodin-Thomazo ${ }^{1}$, F. Malloggi ${ }^{1}$, J. Daillant ${ }^{2}$, R. A. Campbell ${ }^{3,4}$, G. Fragneto ${ }^{3}$, E. B. \\ Watkins $^{3}$, P. Perrin ${ }^{5}$, N. Pantoustier ${ }^{5}$ and P. Guenoun ${ }^{1, a}$ \\ ${ }^{1}$ LIONS, NIMBE, CEA, CNRS, Université Paris-Saclay, CEA Saclay, 91191 Gif-sur-Yvette \\ Cedex, France \\ ${ }^{2}$ Synchrotron Soleil, L' Orme des Merisiers, Saint-Aubin - BP 48, 91192 Gif-sur-Yvette Cedex, \\ France \\ ${ }^{3}$ Institut Laue-Langevin, 71 avenue des Martyrs, 38042 Grenoble, France \\ ${ }^{4}$ Division of Pharmacy and Optometry, University of Manchester, Manchester M13 9PT, UK \\ ${ }^{5}$ Sciences et Ingénierie de la Matière Molle, ESPCI Paris, PSL University, Sorbonne-Université, \\ CNRS, 10 rue Vauquelin, Paris 75005, France
}

\begin{abstract}
Stable stimuli-responsive emulsions between oil and water are formed with an amphiphilic block copolymer bearing polystyrene (PS) and poly(dimethylaminoethyl methacrylate) (PDMAEMA) moieties. Different kinds of emulsions like direct, multiple or inverse ones are reproducibly formed as a function of chemical parameters such as $\mathrm{pH}$ and salt concentration. To test the correlation between the different nature of the emulsion and the conformation of the polymer chain at the interface, neutron reflectometry at the oil/water interface was carried out. An original sample cell was built and the procedure to get reliable results with it on the FIGARO reflectometer at the Institut Laue-Langevin is described. Results show that for direct emulsions, the copolymer is much more extended on the water side than on the oil side. In the case where multiple emulsions are stabilized, the conformation is strongly modified and is compatible with a more equilibrated extension of the chain on both sides. The inverse case shows that the extension in oil is stronger than in water. These results are discussed in term of polymer brushes (charged or neutral) extension with respect to salt addition and hydrophobic interactions.
\end{abstract}

\section{Introduction}

Stimuli-responsive emulsions are interesting structures for vectorizing molecules since a stimulus modifying the emulsion nature [1] can free the molecules which are protected within the emulsion [2]. Stable and reproducible emulsions can be made with polymers [3,4,5], in particular multiple ones formulated with a very precise range of parameters $(\mathrm{pH}$, salt, temperature) $[6,7,8]$. Passing from a direct (oil in water; $\mathrm{O} / \mathrm{W}$ ) to a multiple (water in oil in water; $\mathrm{W} / \mathrm{O} / \mathrm{W}$ ) or to an inverse (water in oil; W/O) emulsion seems to be connected to a particular polymer conformation at the liquid/liquid interface [7]. In the past few years, we developed a system based on the use of a single amphiphilic block copolymer PS- $b$-(PS-stat-PDMAEMA) made of polystyrene (PS) and poly(dimethylaminoethyl methacrylate) (PDMAEMA) moieties and capable of stabilizing in one emulsification step the three types of emulsions, depending on $\mathrm{pH}$ and ionic strength conditions $[2,7]$.

A tool of choice to provide insight into the structure and conformation of layers at fluid interfaces is neutron reflectometry [9], primarily because of the possibility to resolve the extension of the polymer layer (some tens of nanometers) and the use of isotopic contrast variation of the polymer and/or the bulk liquid phases. However, neutron reflectivity measurements at liquid/liquid interface are rarely performed and have been limited so far to few particular examples. Pioneering studies were carried out by Lee et al. [10] and Cosgrove et al. [11] for studying a very thin oil film on top of water in which the molecules of interest are soluble.

a e-mail : patrick.guenoun@cea.fr 
When dealing with thicker oil phases and to get a better control over the oil upper thickness, Zarbakhsh et al. designed an ingenious system by means of a microscopic frozen alkane layer spin coated on a solid crystal and located next to a macroscopic water phase following thawing of the oil phase [12]. More recently Besnard et al. used a preliminary design whose improvement is the basis of this communication [7] and Scoppola et al. used another design consisting of the transmission of neutrons through an oil phase for reaching a buried W/O interface through which water-soluble molecules are extracted [13].

Our cell is designed and optimized for use with systems where the oil phase potentially scatters a large number of neutrons because of the dissolved polymer. A sapphire crystal is used as support of a water phase through which the neutron beam reaches the interface, yet the necessity to match the water phase isotopic contrast with that of the sapphire (see below) means that it is still partly hydrogenous $\left(10 \% \mathrm{v} / \mathrm{v} \mathrm{H}_{2} \mathrm{O}\right.$ in $\left.\mathrm{D}_{2} \mathrm{O}\right)$ and has to be reduced to a minimal thickness. The use of this cell and realization of the experiment has been made possible thanks to use of the high flux FIGARO (Fluid Angles Grazing Incidence ReflectOmeter) [14] reflectometer at the Institut Laue-Langevin (Grenoble, France), which has the possibility to reflect a collimated beam of neutrons down at a horizontal interface. A particularly high flux configuration can be selected thanks to the use of dephased choppers and the instrument being on a continuous reactor source.

In trying to rationalize the very reproducible formation of one type of emulsion in specific $\mathrm{pH}$ and ionic strength conditions, we recently reported preliminary NR results where our cell was used, presenting only one case corresponding to an $\mathrm{O} / \mathrm{W}$ emulsion case [7]. As a result of important improvements of our experimental procedure that here we describe in detail, we present how neutron reflectivity data at the $\mathrm{W} / \mathrm{O}$ interface can be acquired with confidence using our approach after precise normalization and, in the best cases, determination of the water thickness through which the beam transmits. In particular, we show that the density profile of polymer at the oil (toluene)/water interface is noticeably different between the different physicochemical conditions leading to the various kinds of emulsions $(\mathrm{O} / \mathrm{W}, \mathrm{W} / \mathrm{O} / \mathrm{W}, \mathrm{W} / \mathrm{O})$. These results lead to a quantitative determination of the repartition of polymer segments between oil and water and demonstrate a clear link between polymer conformation and nature of the emulsion. The magnitude and algebraic value of the spontaneous curvature is correlated to the emulsion kind.

\section{Methods}

\subsection{Polymers and Emulsion precursor preparation}

$\mathrm{PS}_{40}-b-\left(\mathrm{PS}_{21}\right.$-stat-PDMAEMA 68 , synthesized as described elsewhere [2], was dissolved in deuterated toluene at a concentration of $5 \mathrm{mg} / \mathrm{mL}$. Aqueous phases were made by a 90:10 v/v $\mathrm{D}_{2} \mathrm{O}: \mathrm{H}_{2} \mathrm{O}$ mixture in order to match the scattering length densities of deuterated toluene and sapphire, the solid support material of our home-built experimental cell. The $\mathrm{pH}$ was adjusted with $1 \mathrm{M} \mathrm{HCl}$ solution, and ionic strength was adjusted with $\mathrm{NaCl}$.

\subsection{Neutron reflectivity measurements}

Neutron reflectometry is a technique that is non-invasive and sensitive to light elements, and thanks to the use of isotopic contrast variation it can be used to resolve the composition and structure of organic materials at buried interfaces $[9,15,16]$. A dedicated cell was developed in our lab to be able to study the polymer conformation at water/toluene interface, meeting the following requirements. First, our polymer is soluble only in the oil phase but, when in contact with a water phase, some polymer transfer to the water occurs. However, at the working salinities, most of the polymer stays in oil and to avoid the scattering of the neutron beam by the dissolved polymer, the experimental cell was designed with the specific criterion that the neutron beam transmits from the water phase below the W/O interface. Secondly, as neutrons undergo a strong incoherent scattering by hydrogenous water ( $10 \%$ of our water), the travelling distance of 
the beam through water must be minimized. To comply with these requirements we aimed at stabilizing the largest area $\mathrm{W} / \mathrm{O}$ interface with the lowest water thickness for several hours in order to acquire enough signal. Sapphire was chosen as a material for accommodating the liquid phases since it is nearly transparent to neutrons, it matches the scattering length densities of the 90:10 v/v deuterated/hydrogenous water mixture, and it is very close to that of deuterated toluene, and has the added advantage that it is hydrophilic so water easily spreads on top of it. The cell itself was therefore made of sapphire to make a pool, in which an inner sapphire crystal is immersed. The latter inner sapphire crystal is of dimensions $108 \times 49 \times 10 \mathrm{~mm}^{3}$ (all sapphire parts were bought at Micropierre (Besançon, France)). The pool has external dimensions of 134 $\times 84 \times 25 \mathrm{~mm}^{3}$. A dedicated hole exists in the pool for sucking the water out and tuning the water thickness, and a very smooth sapphire crystal (upper face is polished) was used that was cleaned in a plasma cleaner for $4 \mathrm{~min}$ before each experiment to make it hydrophilic. To adjust the thickness of water we used Teflon spacers put between the bottom of the pool and the sapphire plate (in white in fig. 1).

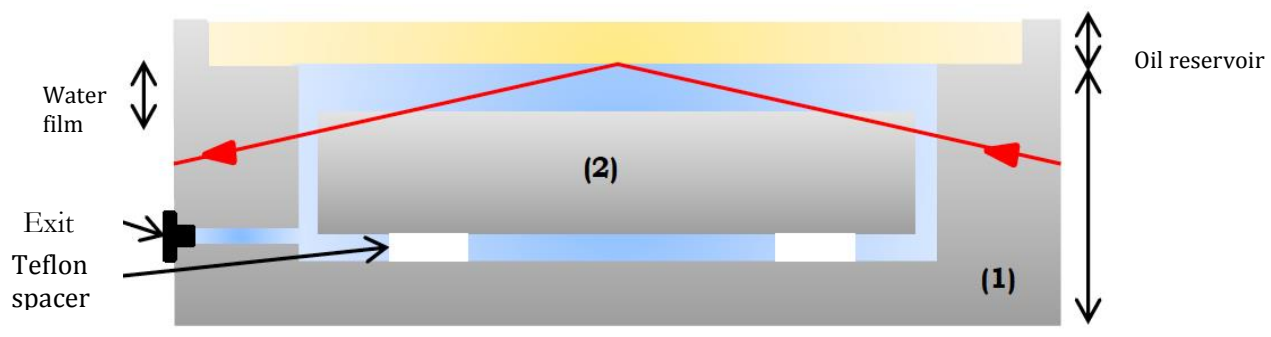

Fig. 1: Schematic diagram of the experimental cell showing (in red) the neutron beam path and its reflection at the W/O interface. (1) indicates the sapphire pool and (2) the inner sapphire crystal.

The exit is located such as no interference with the neutron beam occurs.

After plasma cleaning, the sapphire crystal is placed in the center of the pool and the cell is filled with an excess of water. The toluene polymer solution is then carefully deposited at the surface of water with a syringe. Finally, the excess of water is cautiously removed in order to obtain a flat interface and the desired water thickness (typically 100 micrometers). A critical point is to avoid toluene dewetting on top of the water.

The FIGARO instrument at the ILL (Grenoble, France) was used in a time-of-flight configuration with two angles of incidence $\theta_{1}=0.62^{\circ}$ and $\theta_{2}=2.71^{\circ}$ (both reflection down at the sample). The neutron reflectivity, $\mathrm{R}$, is defined as the number of neutrons in the specular reflection of a sample divided by that in the incident beam, as a function of the wavevector transfer $Q$ normal to the interface, itself calculated as $4 \pi \sin \theta / \lambda$, where $\lambda$ is the wavelength of the incident beam (white beam in the range 2-16 $\AA$ ). The profile of the incident beam as a function of $\mathrm{Q}$ is acquired by a direct beam measurement through as much of the sample cell that the beam will pass during the reflection measurement as possible; any differences in the beam path would require subsequent corrections to the data, as described in certain cases below. During the direct beam measurements, the detector cannot handle the full beam as the neutron intensity is too high, so the signal is reduced in intensity in a wavelength-independent way through the use of an oscillating slit. This results in the need to normalize the reflectivity data by a single scaling factor for each angle, as described below. Note that the values of the scaling factors correspond simply to the proportion of neutrons that transmit through the oscillating slit during the direct beam measurements for each angle, and are not related to any characteristics of the sample. For the 
reflectivity measurements, the signal was integrated on the area detector in a region of interest (ROI) around the specular reflection angle, and the background scattering was measured outside this ROI and subsequently subtracted from the reflectivity data.

We started by measuring air/sapphire reflectivity at $\theta_{1}$ and $\theta_{2}$ in the configuration of fig. 2 a) whose signal was normalized by the direct beam measured as in fig. 2 b). In the latter case, the beam and cell were inclined by $\theta_{1}$ or $\theta_{2}$, respectively (not shown). This procedure led to the final normalized reflectivity data in fig. $2 \mathrm{c}$ ). The normalization process was carried out in 2 stages. First, through knowledge that the reflectivity at the plateau in the region of total reflection below the critical edge $\left(Q_{c}=0.0157 \AA^{-1}\right)$ must be 1 , the data from angle $\theta_{1}$ were scaled in reflectivity accordingly. Secondly, the data from angle $\theta_{2}$ were scaled in reflectivity so that the overlap of the data from the second angle to the first one was correct. Moreover the data were modelled as a single interface with Gaussian roughness between the two bulk media to resolve the scattering length density of the sapphire crystal as $5.76 \times 10^{-6} \AA^{-2}$ (determined from the value of $Q_{c}$ ) and the roughness of the crystal as $7 \AA$ (determined from the falloff of the Fresnel reflectivity). This method of determining the scaling factors through a calibration reflectivity measurement involving air as the ambient medium is the standard one used, and as the values were not related to any aspects of the sample, the factors were applied in the following for normalization of all the subsequent data sets.

The next configuration was closer to the one used in measurements and comprised sapphire/air reflectivity (fig. 2 d) where there was air on both sides of the crystal in the gaps of the pool between the crystal and the cell. Each reflectivity measurement was normalized to the direct beam (fig. 2 e) for which the beam and cell were inclined by $\theta_{1}$ or $\theta_{2}$ in their respective measurements (not shown). In the following step, direct beams were measured through the sapphire crystals immersed in water (a mixture of 90:10 v/v $\mathrm{D}_{2} \mathrm{O} / \mathrm{H}_{2} \mathrm{O}$ ) at the two angles for taking into account the water volume on the sides. The reflectivity data at the sapphire/air interface was then obtained, using the normalization factors previously determined (fig. $2 \mathrm{f}$ ).

a)

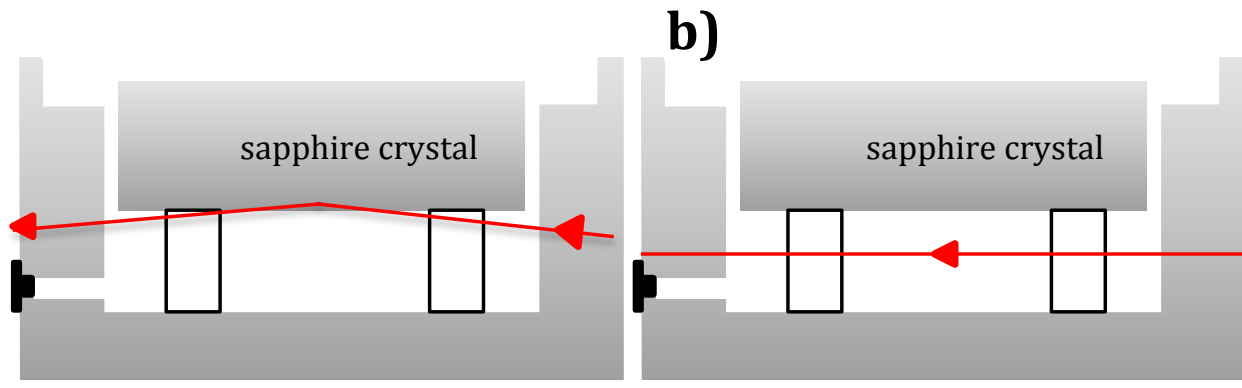

c)

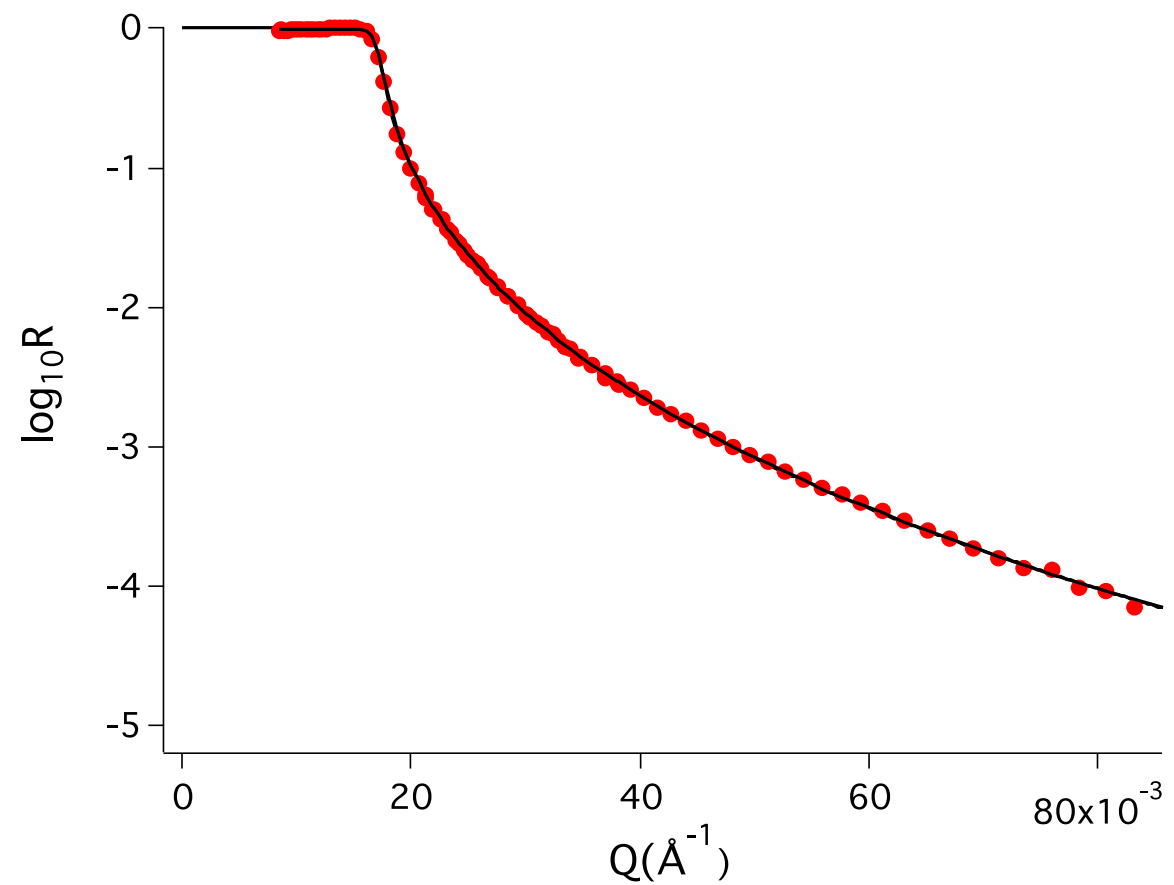



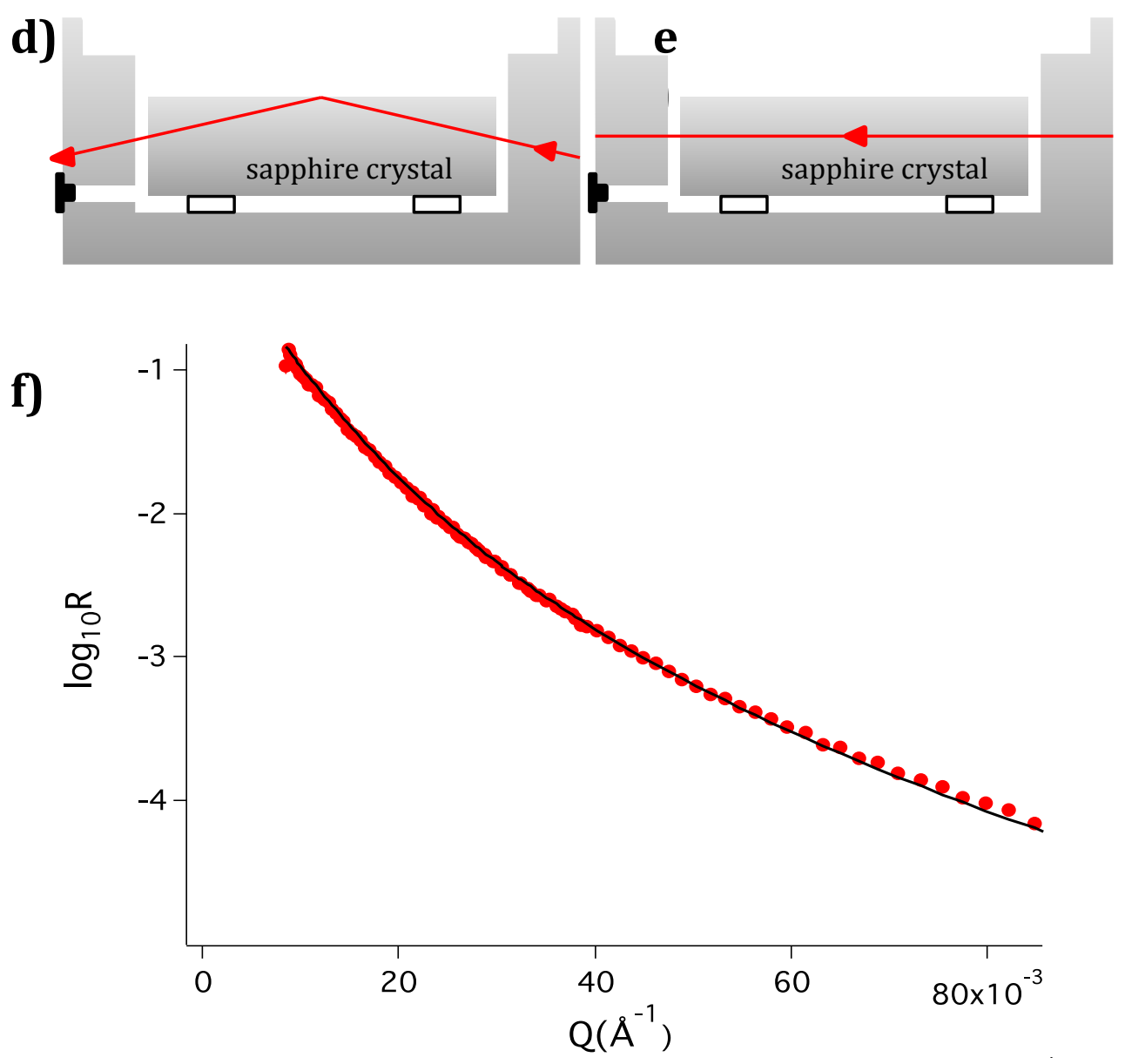

Fig. 2: Schematic diagrams of the configurations of a) reflectivity measurements at the air/sapphire interface and b) associated direct beams, providing the c) air/sapphire reflectivity data after adequate normalization. Schematic diagrams of the configurations of reflectivity measurements at the d) sapphire/air interface and associated direct beams, providing the f) sapphire/air reflectivity data after use of the same normalization factors previously determined.

\subsection{Polymer adsorption}

Some polymer molecules transfer with time from the oil phase to the water phase resulting in adsorption onto the sapphire. This adsorbed polymer contributes to a weak reflectivity signal that will be ordinarily convoluted with that of the W/O interface (fig. 3 a). In order to calibrate this contribution to the measured reflectivity data, a measurement was performed by making the water thickness much thicker (i.e. several $\mathrm{mm}$ ) than during measurements of the W/O interface (i.e. removal of the signal from W/O interface). The water/oil interface was then high enough such that no neutrons were incident on this interface. The only signal measured was therefore due to the adsorbed polymer at the sapphire/water interface. This signal, measured at angles $\theta_{1}$ and $\theta_{2}$, is shown in fig. 3 b). This contribution was checked to be independent of physicochemical conditions used afterwards and was taken into account in the analysis by subtracting the corresponding reflectivity. The corresponding adsorbed layer is found to be of a thickness of $110 \pm 30 \AA$ with a scattering length density of $5.4 \pm 0.2 \times 10^{-6} \AA^{-2}$ and a roughness of $80 \pm 30 \AA$.

\section{a)}

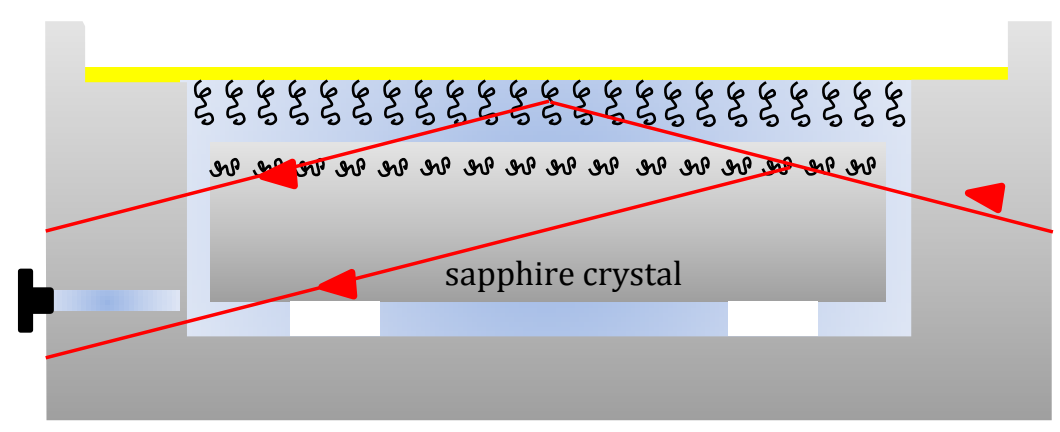




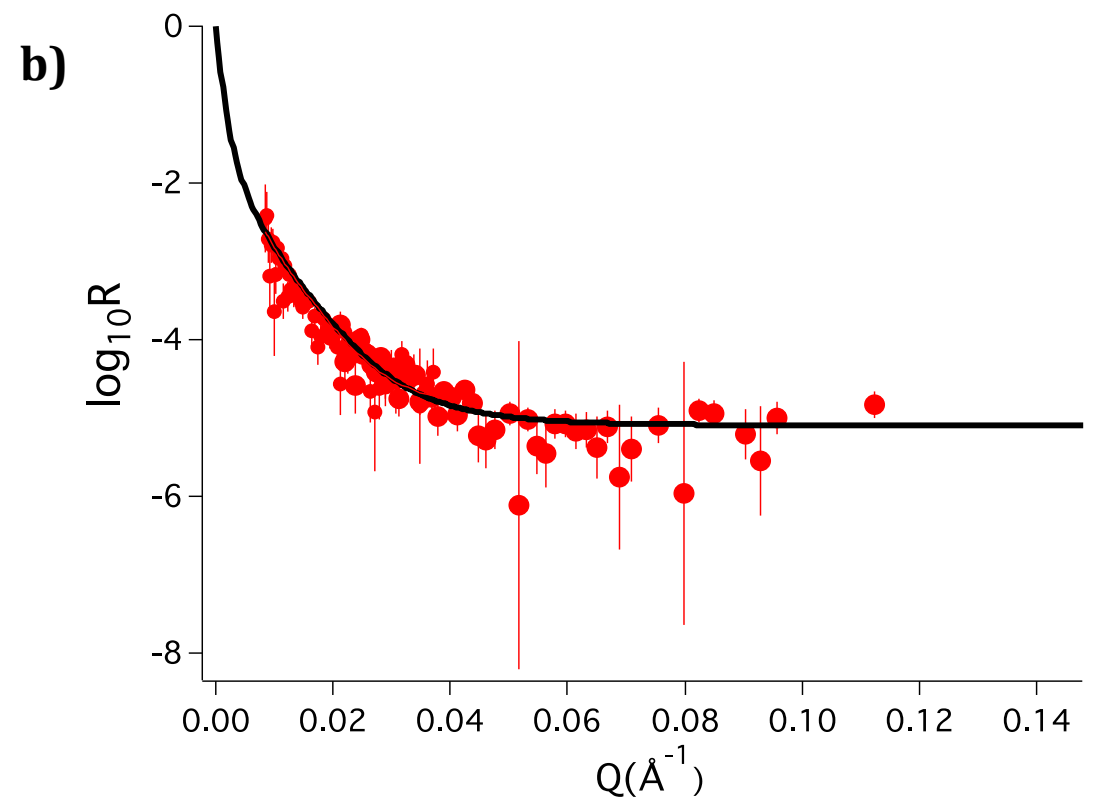

Fig. 3: a) Schematic diagram of the convoluted measurements at the W/O interface with that of adsorbed polymer layer at the sapphire/water interface, b) the reflectivity data that were measured to calibrate this contribution at the sapphire/water interface alone. This was made by measuring only the latter interface by thickening the water layer by a large amount.

\subsection{Transmission measurement}

Direct beams were measured through the sapphire crystal and the water present on both sides of it (one for each configuration of the collimation slits which were different for $\theta_{1}$ and $\theta_{2}$ ), while the reflected beam also transmitted through the partly hydrogenous water above the inner sapphire crystal over a distance $L$, which depends on the incidence angle and the water thickness e: $L_{1(2)}=2 \mathrm{e} / \sin \theta_{1(2)}$. This value was typically on the order of $1-2 \mathrm{~cm}$, and its significance meant that initially the reflectivity data acquired at the two different incident angles did not properly overlap. In order to calibrate this effect, the transmission of the neutron beam through different thicknesses of water was measured independently with quartz cells of different thicknesses. This transmission function depends on the wavelength (and neutron energy), and therefore it affects to different extent the wavevector $\mathrm{Q}$, and the reflectivity data from different incident angles. We checked that for a distance $L_{1(2)}$ in centimeters, the transmission is equal to $T_{1 \mathrm{~cm}}(\mathrm{Q})$, with $\mathrm{T}_{1 \mathrm{~cm}}$ the transmission obtained for a $1-\mathrm{cm}$ thick cell. For both angles of measurement, each dataset was then re-normalized by the function $\mathrm{T}_{1 \mathrm{~cm}}(\mathrm{Q})^{\mathrm{L}}$ (each reflectivity was divided by $\mathrm{T}_{1 \mathrm{~cm}}(\mathrm{Q})^{\mathrm{L}}$, which provides the true reflectivity, see fig. 4) where $L$ is $L_{1(2)}$ for the angle $\theta_{1(2)}$. The e value was determined by the requirement that the data recorded at the two incident angles must superimpose in the overlap region of wavevectors (fig. 4). It is of course important to make sure that the water thickness is always much larger than the polymer layer thickness (on the order of $10 \mathrm{~nm})$

In some preliminary experiments when using configuration of fig. 2 , the water film above the sapphire crystal was evidently thinning over time for a period that was comparable to the measurement time. As a result, in later experiments we used another method of filling to avoid this effect by removing the lower Teflon spacers and avoiding pouring toluene directly into the uppermost compartment while the thin water film had already been prepared (fig. 1). This procedure eliminated the possibility of mechanical perturbations to the system that enhanced the thinning process. In the results presented here, we retained only the measurements where it was sure that a thick enough water layer was present, either by transmission determination (like in fig. 
4 where the uncorrected data from $\theta_{1}$ and $\theta_{2}$ are distinct enough so as to determine $\mathrm{L}$ through application of the correction to make the curves overlap) or by checking that a stable reflectivity signal was measured over time. After each experiment, it was also checked visually that water remained in contact with the sapphire crystal.

\section{a)}

b)
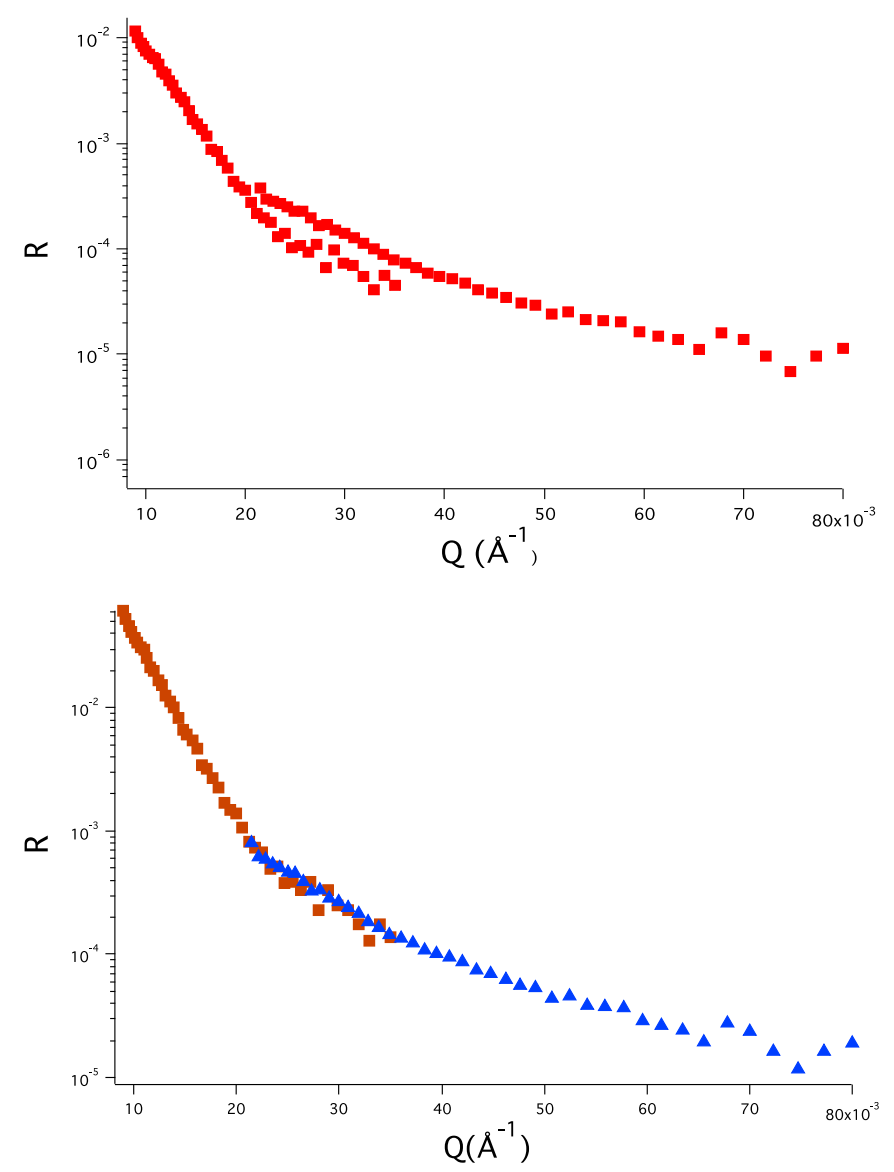

Fig. 4: Example of reflectivity data of a polymer layer at the W/O interface a) uncorrected for the transmission through water, b) after correction by the transmission factor determined by curve overlap.

\subsection{Data treatment and analysis}

After conversion of the data from the area detector of of FIGARO by the COSMOS program in the LAMP software [17], absolute reflectivity data (neutron intensity normalized by the direct beam and taking into account the normalization factors appropriate for the measurements) corrected for background scattering were obtained as a function of wavevector Q. The Motofit software [18] was used to model and fit the absolute reflectivity data. It is a package that provides the least squares fitting of neutron reflectivity data, using an Abeles Matrix formalism or Parratt recursion formula for specular reflection from stratified media [19]. Each layer (in number $n$ ) is characterized by its thickness and scattering length density to which must be added $n+1$ roughnesses.

\section{Results}

\subsection{Comparison of reflectivities}

In the following we will present three examples corresponding to conditions where direct, multiple and inverse emulsions are formed. Reflectivites corresponding to the conditions 
described above are shown in fig. 5. They are markedly different indicating, before any data quantitative interpretation, that different chain conformations occur in each case. To enhance differences between data and corresponding fits, data are presented in a $R^{4}$ vs. Q representation. Note that ideally we may have included a water/toluene reflectivity measurement as reference in this figure, but the measurement was not achieved due to the high curvature of the interface as a result of the relatively high surface tension.

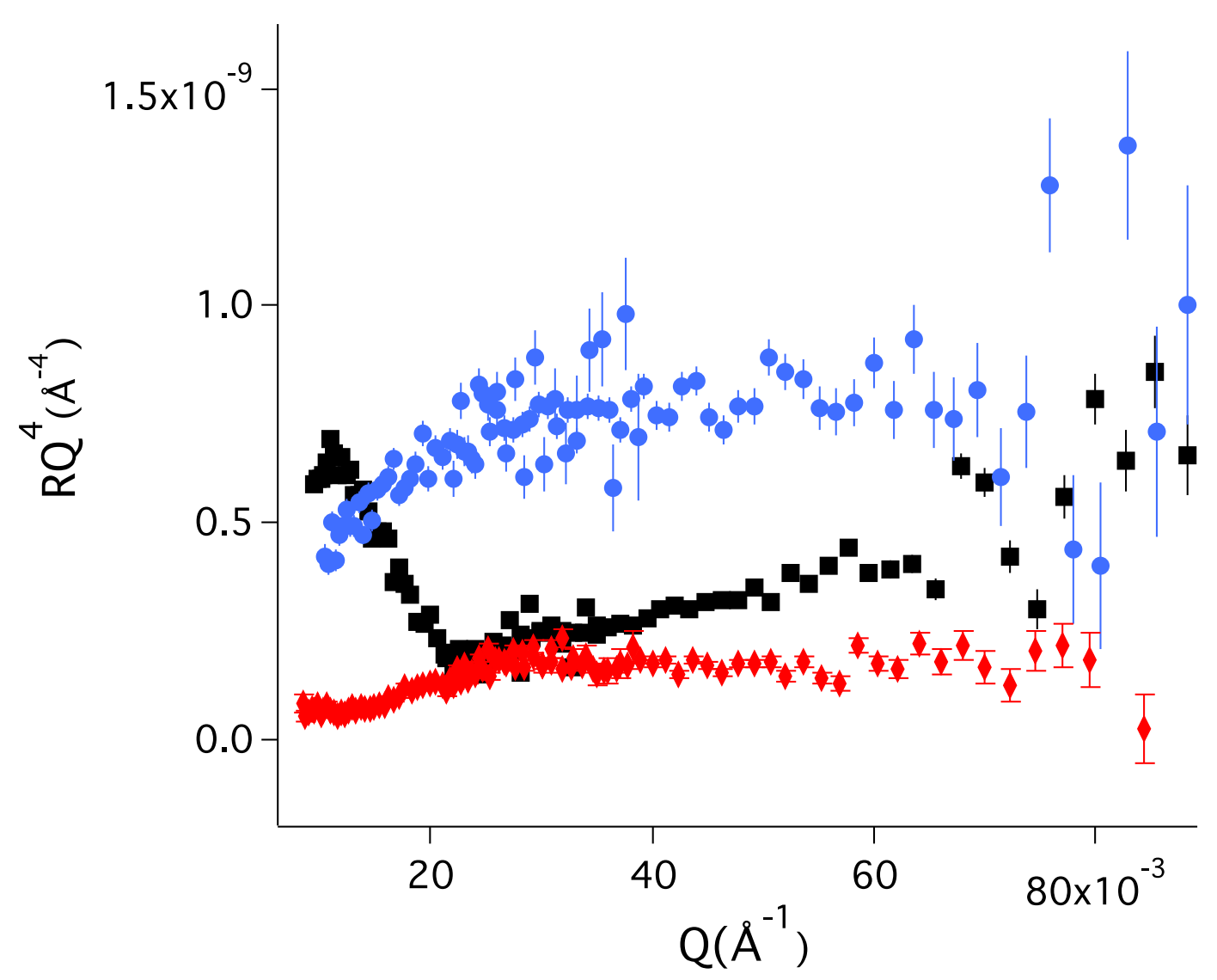

Fig. 5: Comparison of reflectivity data for the different cases: direct (black squares), multiple (blue circles) and inverse (red diamonds) emulsions.

\subsection{Direct emulsion case}

The direct emulsion case is obtained after contact for $24 \mathrm{~h}$ of a toluene polymer solution with a water $\mathrm{NaCl}$ solution of $0.2 \mathrm{M}$ set at $\mathrm{pH} 1$ by addition of $\mathrm{HCl}$. After this time, emulsification by Ultraturrax for 40 s readily forms $\mathrm{O} / \mathrm{W}$ emulsions, as shown by confocal images where a hydrophobic dye is dissolved in oil prior to mixing [7]. Neutron reflectivity measurements were acquired in the same conditions except that the W/O interface was studied just after contact of phases and without any mixing. Polymer transfer to the water phase is known to occur just after contact and its kinetics was measured by UV-visible spectrophotometry with time.[20] Reflectivity data acquired after equilibrium are shown in fig. 6 along with the best fit that could 
be obtained using a one-layer model. The resolution was taken to be $\Delta \mathrm{Q} / \mathrm{Q}=8.6 \%$, which resulted from the chopper and slit settings used experimentally. In such a fit, 6 parameters are to be determined, i.e. the background, the layer thickness, the water phase scattering layer density, the layer scattering length density and the roughness at each interface of the layer (with the bottom water and the top oil). This one-layer fit is clearly unsatisfactory for any set of parameters. Note that, in this direct case as in the others, it was not helpful to include in the fit a water sublayer including some transferred bulk copolymer involving modification of the scattering length density from the nominal value of $5.76 \times 10^{-6} \AA^{-2}$.

A two-layer fit was also undertaken, and it provides very good agreement with the data (fig. 6). The model parameters are $\mathrm{L}_{\mathrm{Ws}}=185 \pm 4 \AA, \mathrm{L}_{\mathrm{OS}}=73 \pm 5 \AA, \mathrm{Nb}_{\mathrm{ws}}=4.07 \pm 0.02 \times 10^{-6} \AA^{-2}$, and $\mathrm{Nb}_{\text {os }}=4.35 \pm 0.04 \times 10^{-6} \AA^{-2}$, where $\mathrm{L}_{\mathrm{WS}(\mathrm{OS})}$ denotes the extension of the layer on the water (oil) side and $\mathrm{Nb}_{\mathrm{WS}(\mathrm{OS})}$ denotes the scattering length density on the water (oil) side.

The distinction of two separated layers is compatible with a polymer monolayer whose hydrophilic block extends as a charged brush in water and hydrophobic block as a neutral brush in oil. A sketch of these models for the different configurations studied here is proposed in fig. 7. This model involves a useful constraint in the data fitting of requiring a unique block area per chain, $\sigma$, calculated with parameters of each layer. This area can be defined as: $\sigma=\mathrm{N}_{\mathrm{PM}(\mathrm{PS})} \mathrm{V}_{\mathrm{PM}(\mathrm{PS})} /\left[\mathrm{L}_{\mathrm{WS}(\mathrm{OS})} \varphi_{\mathrm{PM}(\mathrm{PS})}\right]$, where $\mathrm{N}_{\mathrm{PM}(\mathrm{PS})}$ is the number of segments on the hydrophilic (hydrophobic) side, $\mathrm{v}_{\mathrm{PM}(\mathrm{PS})}$ the specific volume of the segments on the hydrophilic (hydrophobic) side, $\varphi_{\mathrm{PM}(\mathrm{PS})}$ the volume fraction of segments on the hydrophilic (hydrophobic) side, and $\sigma_{\mathrm{PM}}$ $=\sigma_{\mathrm{PS}}$. The suffix PM stands for the complex hydrophilic block made of PS and PDMAEMA where the latter are fully ionized at the working $\mathrm{pH}$ and the suffix PS for poly(styrene).

The values of $\mathrm{L}_{\mathrm{Ws}(\mathrm{OS})}$ and $\varphi_{\mathrm{PM}(\mathrm{PS})}=\left(\mathrm{Nb}_{\mathrm{Ws}(\mathrm{OS})}-\mathrm{Nb}_{\text {water(toluene) }}\right) /\left(\mathrm{Nb}_{\mathrm{PM}(\mathrm{PS})}-\mathrm{Nb}_{\text {water(toluene) }}\right)$ are determined by the fit (see Table 1 for numerical values) and are constrained to provide consistent values on both sides. The specific volume of PS and PDMAEMA segments are $164 \AA^{3}$ and $225 \AA^{3}$ [21]. For the statistical block, we made the assumption of an $\mathrm{N}$-average specific volume and an $\mathrm{N}$-average scattering length density for a segment of the hydrophilic block (PDMAEMA-s-PS).

Thanks to this constraint, the confidence interval of the parameter fit can be narrowed to $\mathrm{L}_{\mathrm{WS}}=183 \pm 2 \AA, \mathrm{L}_{\mathrm{OS}}=74 \pm 2 \AA, \mathrm{Nb}_{\mathrm{WS}}=4.07 \pm 0.02 \times 10^{-6} \AA^{-2}$, and $\mathrm{Nb}_{\mathrm{OS}}=4.33 \pm 0.02 \times 10^{-6} \AA^{-2}$ leading to a common molecular area of $\sigma=282 \pm 2 \AA^{2}$ (fig. 6). 


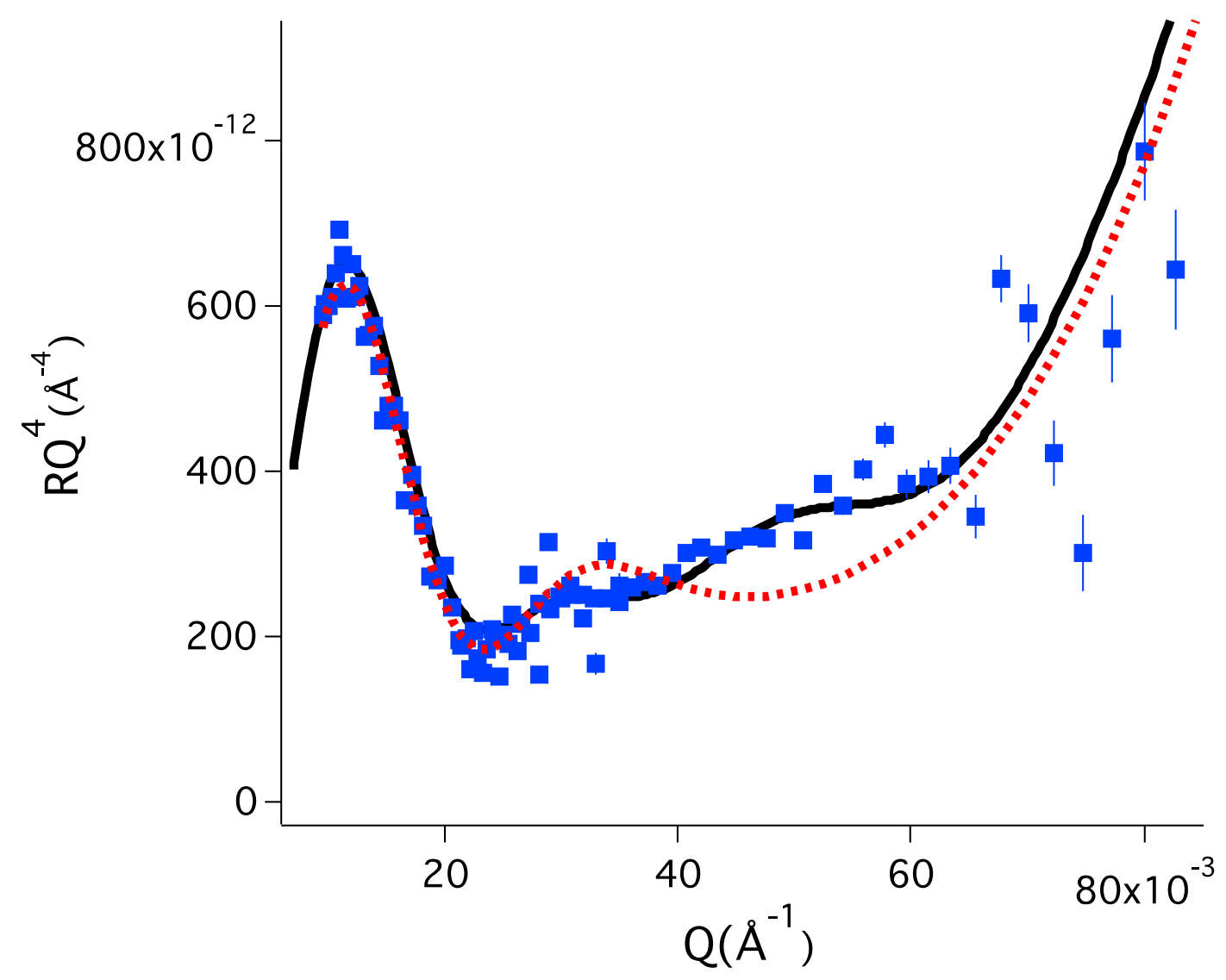

Fig. 6: Reflectivity data for the direct emulsion case presented in a representation where reflectivity is multiplied by $\mathrm{Q}^{4}$ to enhance visually the features that are to be reproduced by the model. Unsatisfactory fit (dashed curve) with a one-layer model. Satisfactory fit involving twolayers of different polymer densities (full curve)

\begin{tabular}{|l|l|l|l|l|}
\hline Monomers & styrene & $\begin{array}{l}\text { dimethylaminoethyl } \\
\text { methacrylate }\end{array}$ & $\begin{array}{l}\text { Deuterated } \\
\text { toluene }\end{array}$ & $\begin{array}{l}90 / 10 \\
\text { deuterated water }\end{array}$ \\
\hline $\mathrm{Nb}\left(10^{-6} \AA^{-2}\right)$ & 1.43 & 1.03 & 5.67 & 5.76 \\
\hline
\end{tabular}

Table1

The volume fraction occupied by the polymer in the layer on the water side is $35 \%$, whereas it is $32 \%$ on the toluene side. The extension on the water side is $183 \AA$, much bigger than on the oil side where it is $74 \AA$. Both values are compatible with brushes extensions on both sides (the square root of the area per chain is smaller than the gyration radius of the PS chain on the hydrophobic side). These values will be discussed in section 4 below. 

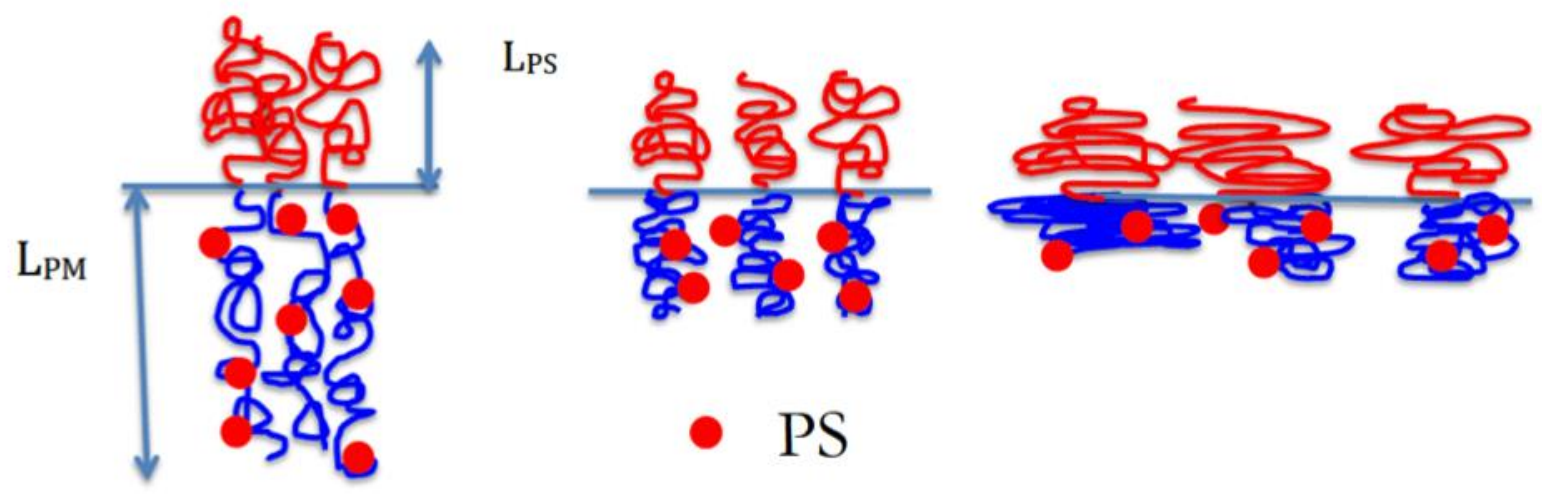

Fig. 7: Sketch of the models with different polymer conformations in agreement with values obtained from the fits. From left to right are presented the direct emulsion case with two extended charged and neutral brushes on the lower (upper) water (oil) side, the multiple emulsion case where both extensions of chains are roughly similar on each side, keeping a low molecular area, and the inverse emulsion case where the water side is more contracted than the oil side at a larger molecular area. Red balls are used to represent some of the PS monomers included in the hydrophilic (PS-stat-PDMAEMA) block: in the direct emulsion case these PS monomers are assumed to have very weak interactions between themselves; in the multiple and inverse emulsion cases they act as stickers to make intra- or inter-molecular bridges

\subsection{Multiple emulsion}

Likewise, a situation of multiple emulsions was mimicked by contacting a polymer toluene solution with a water phase at $\mathrm{pH} 1$ but with a $\mathrm{NaCl}$ salt concentration of $1 \mathrm{M}$. After a period of equilibration, the reflectivity was recorded and is shown in fig. 8. The fit with one-layer leads to a thickness of roughly $120 \AA$ with a roughness on the same order of magnitude, i.e. a very poorly defined layer with no distinctive feature. An attempt was made to distinguish two layers by imposing on the toluene side a PS brush of similar extension to that of the direct emulsion case. The fit was also constrained by imposing again an equal area per block on each side as calculated from the fit parameters. This two-layer model can be applied successfully and leads to two layers of roughly identical thickness $\left(\mathrm{L}_{\mathrm{WS}}=60 \pm 5 \AA, \mathrm{L}_{\mathrm{OS}}=60 \pm 5 \AA\right)$ and identical molecular area of $\sigma=460 \pm 70 \AA^{2}$ calculated on the water and oil sides. This analysis is then in agreement with an equal extension of segments on both sides of the interface. The volume fraction on the oil side is $15 \pm 5 \%$ and on the water side $75 \pm 5 \%$, because of the reduction in extension at a low molecular area. 


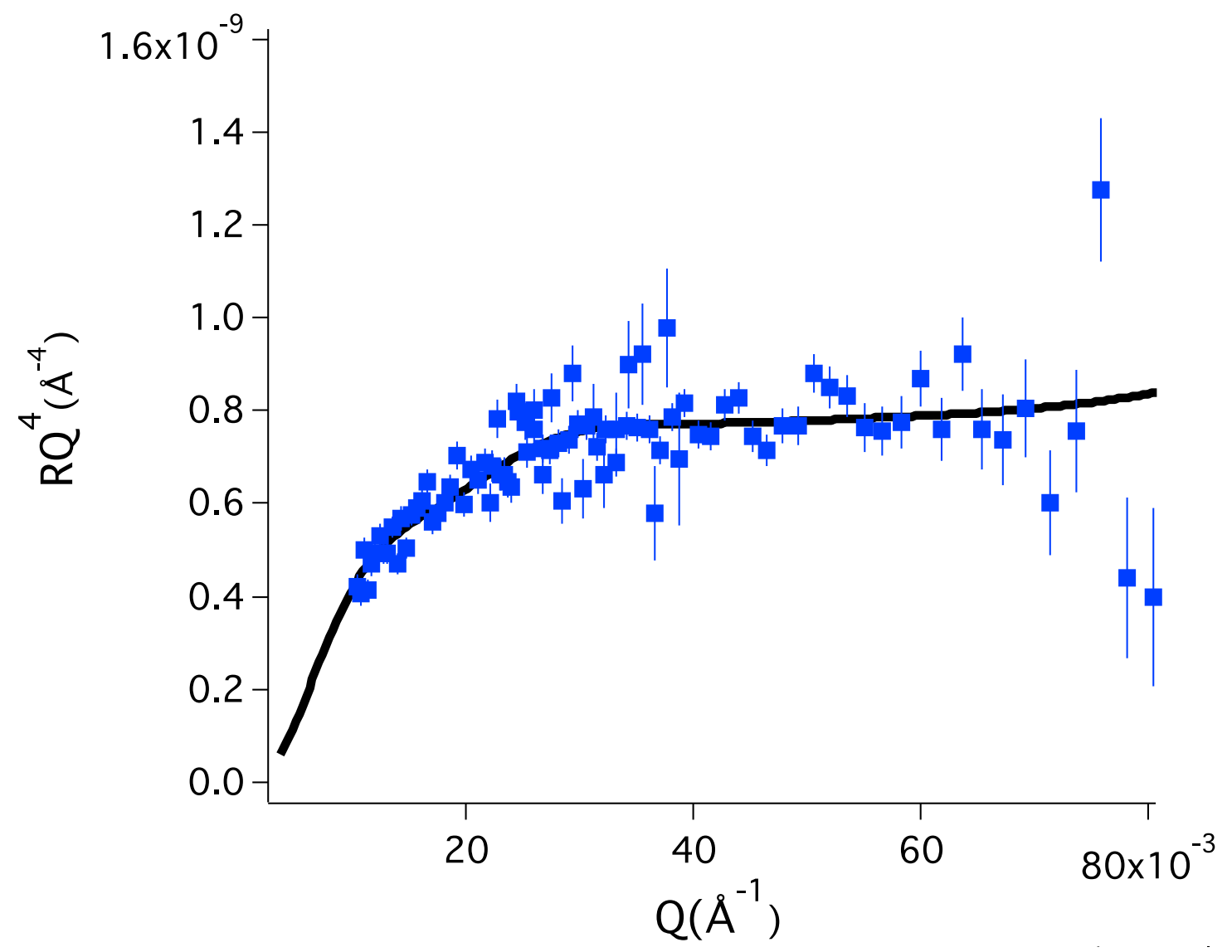

Fig. 8: Reflectivity data for the multiple emulsion case (see text for sample details) and corresponding two-layer fit with an equal block area on both sides $\left(\mathrm{L}_{\mathrm{wS}}=\mathrm{L}_{\mathrm{OS}}=60 \AA\right)$.

\subsection{Inverse emulsion}

The inverse emulsion case was obtained by contacting a polymer solution at $\mathrm{pH} 1$ with a water phase containing $2.5 \mathrm{M} \mathrm{NaCl}$. After equilibration, although the set-up without Teflon ${ }^{\circledR}$ spacers was used to keep a water layer thick enough, the water final thickness could not be determined in this configuration, being probably still too small for inducing a noticeable change in the reflectivities measured at the two different angles. However a stable reflectivity signal from a position of several tens of micrometers above the sapphire plate was recorded.

A one-layer model gives a satisfactory fit with a $78 \AA$ layer that has a scattering length density of $4.8 \times 10^{-6} \AA^{-2}$ and $40 \AA$ roughness. It was again tried to apply a two-layer model with equality of block areas on both sides and the result is shown in fig. 9 which indicates an equal molecular area of $2000 \AA^{2}$. The extension of chains on the water side $(30 \pm 5 \AA)$ is now smaller than on the oil side $(50 \pm 5 \AA)$. The volume fraction on the oil side is of $6 \%$ and of $30 \%$ on the water side. 


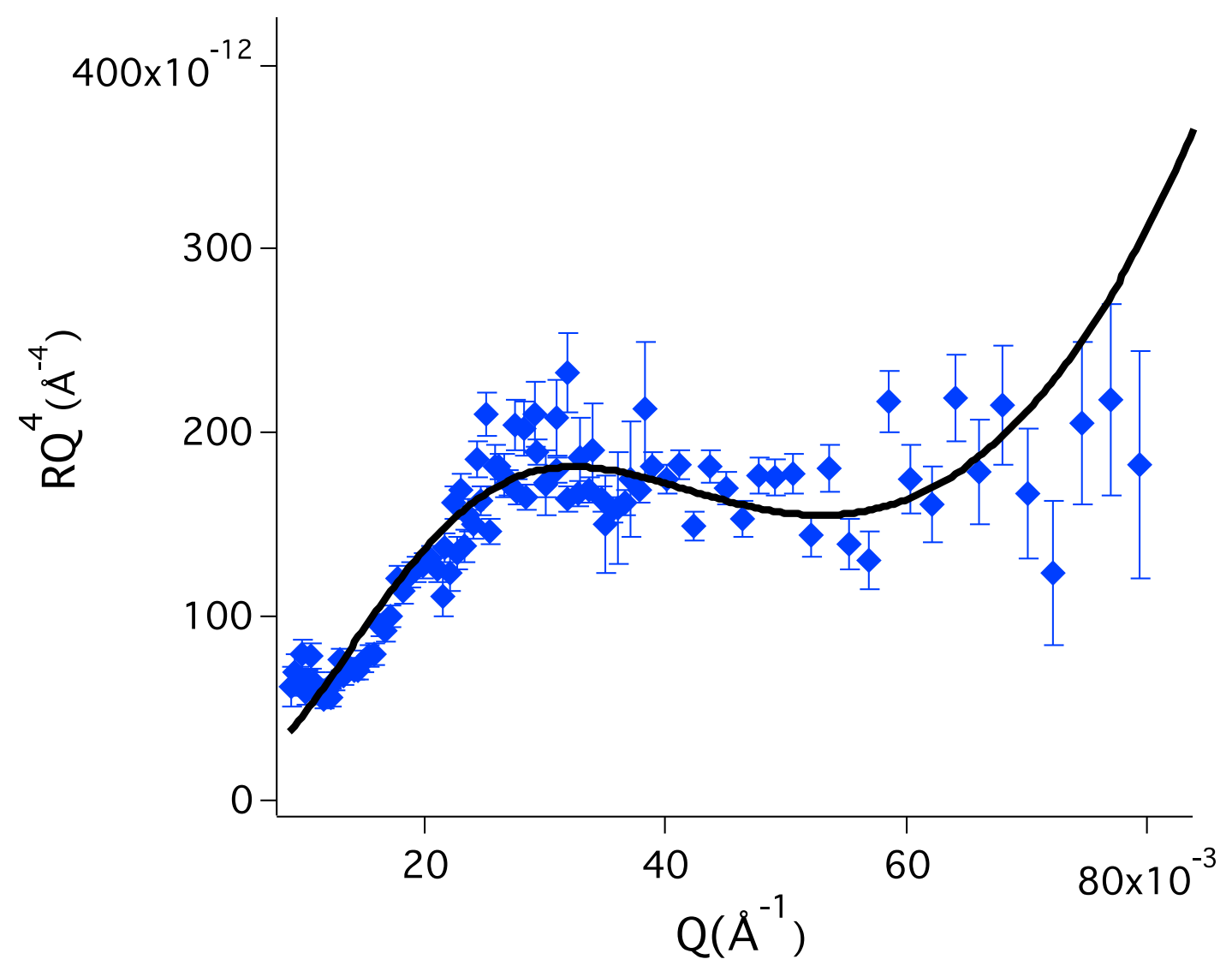

Fig. 9: Reflectivity data of the inverse emulsion case (see text for sample details) and a corresponding two-layer fit with $\mathrm{L}_{\mathrm{WS}}=30 \AA$ and $\mathrm{L}_{\mathrm{OS}}=50 \AA$.

\section{Discussion}

We discuss first about the consistency of our fit interpretations in terms of chain extensions. In the direct emulsion case, the PS brush extension on the oil side can be compared to data from Auroy et al. [22] where the PS brush extension was measured also by neutron reflectometry for a deuterated PS of molecular weight $69,000 \mathrm{~g} / \mathrm{mol}$ at different molecular areas. An extension of $595 \AA$ was found for a molecular area of $\sigma=1260 \AA^{2}$ and a number of segments of $\mathrm{N}=616$. Through application of the scaling of neutral brush extension, which goes as $\mathrm{N}^{1} \sigma^{-1 / 3}$ [23], one could consider, for our case of $\mathrm{N}=40$ and $\sigma=282 \AA^{2}$, an extension of about $64 \pm 3 \AA$, which compared to our finding of $74 \pm 2 \AA$ a very satisfactory match although a little bit over-extended in our case.

On the water side, the osmotic result of Pincus for a charged brush [24] is more difficult to apply since it writes $\mathrm{L}=\mathrm{N}_{\mathrm{PM}} \mathrm{f}^{1 / 2}$ a for a homogeneous charged brush of monomer periodicity a and Manning factor $\mathrm{f}$ [25], which is quite different from our case where one third of PS hydrophobic monomers are statistically mixed with two thirds of charged ones of PDMAEMA. By using a renormalized Manning factor of 0.5 (1/3 of charged monomers are now more spaced from each other due to the PS monomers) instead of the usual Manning factor $f=2.5 / 7=0.36(7 \AA$ is the Bjerrum length in water), a charged extension of $157 \AA$ is calculated, which is somewhat smaller than the determined value of $185 \AA$. It is important to remark that the inner free counterions concentration writes as $\mathrm{fN}_{\mathrm{PDMAEMA}} / \mathrm{L}_{\mathrm{PM}} \sigma$ and is $\cong 1 \mathrm{M}$, much higher than the external salt concentration of $0.3 \mathrm{M}$, justifying the use of the $\mathrm{L}=\mathrm{N}_{\mathrm{PM}} \mathrm{f}^{1 / 2}$ a formula. Another effect can be put forward to explain the rather strong extension of $183 \pm 2 \AA$ : over-extension was demonstrated for charged brushes of small molecular areas (typically less than $2000 \AA^{2}$ [26]), exhibiting stronger 
extensions than the Pincus formula. This is due to potentially two effects: first the volume left to accommodate counterions is limited at small molecular areas by the finite size of chains, forcing the chains to extend more [27]; secondly the Manning factor can be modified in such dense media [28].

When salinity increases like in the multiple emulsion case, the charged brush is shown to shrink quite abruptly from $185 \AA$ to about $60 \AA$, an effect which cannot be explained only by additional screening of ions. Here, above some threshold in screening, we suggest that the PS monomers are now able to act as stickers to minimize hydrophobic interactions, causing a chain 'collapse' as observed when multivalent ions are added to charged brushes and are shown to bridge chains together [29]. On the PS-oil side, the PS brush merely contracts as an effect of molecular area increase.

When the salinity increases more (inverse emulsion case), much less polymer is adsorbed leading to an increase of the molecular area up to $2000 \AA^{2}$. The contraction continues on both sides but to a larger extent on the water side because of the even larger salinity.

Our neutron reflectivity measurements at the W/O interface clearly show changes in interfacial behaviors correlated with the bulk behavior (emulsion nature). The latter nature was shown recently not to be predicted by the Bancroft principle [7], which relates the surfactant solubility to the emulsion nature. In the presented case, the copolymer is mostly oil-soluble with a transfer to water (by contact with oil) that clearly diminishes as one goes from direct emulsion to inverse emulsions conditions. Another possibility in the interpretation of our results is to connect the emulsion nature to the preferential curvature of the polymer film as predicted by Kabalnov and Wennerström from considerations about the coalescence of domains [30]. In the direct $(\mathrm{O} / \mathrm{W})$ case, as it is known from the ethoxylated surfactant case $\left(\mathrm{C}_{\mathrm{i}} \mathrm{E}_{\mathrm{j}}\right)$, the spontaneous curvature of our polymer film is turned towards oil, as it was shown by cryoTEM pictures evidencing spherical structures in water, the core of which is filled by oil (as proved by small angle neutron scattering data) [7]. This is confirmed by the neutrons reflectometry results at the W/O interface presented here where the hydrophilic side of the polymer is clearly more extended than the hydrophobic side. For the inverse emulsion case (W/O), one would expect the opposite result from the ethoxylated reciprocal case where spontaneous curvature is turned towards water at high temperatures. Our results confirm this tendency, tending to a thin dense hydrophilic layer below a more dilute hydrophobic layer on the oil side.

For the multiple emulsion case, no indication can be found in the literature since no stable multiple emulsions can be found for usual surfactant systems, like $C_{i} E_{j}$ for instance. Here we show for the first time that the block copolymer split segments in a rather equal way on both sides of the interface, pointing to a spontaneous curvature close to zero at the interface.

The exceptional stability and very high reproducibility of formation - including for multiple emulsions - is consistent with a specific polymer conformation corresponding to a well-defined kind of emulsion. However, it is still unclear what is the kinetics and pathway of formation leading to multiple architectures in a single step. The absence of observed multiple emulsions of the $\mathrm{O} / \mathrm{W} / \mathrm{O}$ kind with our system, while $\mathrm{W} / \mathrm{O} / \mathrm{W}$ ones are readily obtained, probably indicates more a slight asymmetry between bulk phases than a noticeable asymmetry in the polymer interfacial conformation.

\section{Conclusion}

We have demonstrated in this work that neutron reflectivity measurements at the liquid/liquid interface, although needing careful calibration procedures, are an invaluable tool for exploring complex polymer conformations at the water/oil interface. Such an advance was possible thanks to optimization of a sample cell involving a macroscopic oil phase that is suited to measurements at the water/oil interface in a reflection down configuration while exploiting the high flux FIGARO reflectometer at the ILL. The data interpretation is another complex procedure that needs careful consistency checks. Such a procedure was attempted and made possible here because a model was constructed for refinement of the parameters. Experimental challenges in the future consist of better control over the wetting states of the substrates in order to reach a 
better defined water thickness. Even so, it was possible as a result of the technical advances described in this work for us to compare the coverage and extension in both the oil and water phases of polymers present in direct, multiple and inverse emulsions for the first time.

\section{Acknowledgements}

The authors thank the Institut Laue-Langevin for award of beamtime (doi: 10.5291/ILL-DATA. 9-11-1748) and use of support laboratories within the PSCM facilities.

\section{Author contribution statement}

MP and NBT synthesized the polymer, prepared the experiment by careful sample cell testing, ran the experiment and analyzed data, FM helped preparing the sample cell and ran the experiment, JD contributed to data analysis and report writing, RAC made the FIGARO instrument functional, supervized the data acquisition and normalization, advised on the data analysis and assisted with paper writing, GF and EBW assisted during the neutron experiments, NP and PP supervized the synthesis and general work and PG supervized the work, ran the experiment, analyzed data and wrote the manuscript.

1. B. P. Binks, Colloids Surf. Physicochem. Eng. Asp. 71, 167-172 (1993).

2. L. Besnard, F. Marchal, J.F. Paredes, J. Daillant, N. Pantoustier, P. Perrin, P. Guenoun, Adv. Mater. 25, 2844-2848 (2013).

${ }^{3}$ A.M. Mathur, B. Drescher, A.B. Scranton, J. Klier, Nature 392, 367-370 (1998).

4 P. Perrin, N. Monfreux, F. Lafuma, Colloid Polym. Sci. 277, 89-94 (1999).

5 P. Perrin, L. Porcar, C. Tribet, Polym. Int. 52, 465-470 (2003).

${ }^{6}$ L. Hong, G. Sun, J. Cai, T. Ngai, Langmuir 28, 2332-2336 (2012).

${ }^{7}$ L. Besnard, L. M. Protat, F. Malloggi, J. Daillant, F. Cousin, N. Pantoustier, P. Guenoun, P. Perrin, Soft Matter 10, 7073-7087 (2014).

${ }^{8}$ Q. Chen, X. Deng, Z. An, Macromol. Rapid Commun. 35, 1148-1152 (2014).

${ }^{9}$ L. Braun, M. Uhlig, R. von Klitzing, R. A. Campbell. Adv. Colloid Interface Sci. 147, 130148 (2017).

10 L.T. Lee, D. Langevin, B. Farnoux, Phys. Rev. Lett. 67, 2678-2681 (1991).

11 T. Cosgrove, J.S. Phipps, R.M. Richardson, Colloids Surf. 62, 199-206 (1992).

12 A. Zarbakhsh, J. Bowers, J.R.P. Webster, Meas. Sci. Technol. 10, 738 (1999) ; A.

Zarbakhsh, J.R.P. Webster, J. Eames, Langmuir 25, 3953-3956 (2009).

13 E. Scoppola, E. Watkins, G. Li Destri, L. Porcar, R. A. Campbell, O. Konovalov,

G. Fragneto, O. Diat, Phys. Chem. Chem. Phys. 17, 15093-15097 (2015) ; E. Scoppola, E.

B. Watkins, R. A. Campbell, O. Konovalov, L. Girard, J.F. Dufrêche, G. Ferru, G. Fragneto, O. Diat Angew. Chem. 55, 9326 (2016).

14 R. A. Campbell, H. P. Wacklin, I. Sutton, R. Cubitt, G. Fragneto. Eur. Phys. J. Plus 126, 107, (2011)

15 J. R., Lu, R. K. Thomas, J. Penfold, Adv. Colloid Interface Sci., 84, 143-304 (2000).

16 T. Narayanan, H. Wacklin, O. Konovalov, R. Lund, Crystallogr. Rev., , 23, 160-226

(2017).

17 P. Gutfreund, T. Saerbeck, M.A. Gonzalez, E. Pellegrini, M. Laver, C. Dewhurst, R. Cubitt, J. Appl. Crystallogr. 51, 606-615 (2018)

18 A. Nelson, J. Appl. Crystallogr., 39, 273. (2006).

19 L. G. Parratt, Phys. Rev., 95, 359. (1954) 
${ }^{20}$ Lucie Besnard, PhD thesis (Université P. et M. Curie, 2013), fig. 7, page 95.

${ }^{21}$ H. Lee, V. Tsouris, Y. Lim, R. Mustafa, J. Choi, Y. H. Choi, H.-W. Park, M. Meron, B. Lin, Y.-Y. Won, Soft Matter, 10, 3771, (2014)

22 P. Auroy, Y. Mir, L. Auvray, Physical Review Letters, 69, 93 (1992).

${ }^{23}$ A. Halperin, M. Tirrell and T. P. Lodge, Advances in Polymer Science, vol. 100 Springer (1992)

24 P. Pincus, Macromolecules 24, 2912 (1991).

25 G.S. Manning, J. Chem. Phys., 51, 924-933, (1969).

26 G. Romet-Lemonne, J. Daillant, P. Guenoun, J. Yang, J.W. Mays, Phys. Rev. Lett. 93, 148301 (2004).

27 A. Naji, R. R. Netz, C. Seidel, Eur. Phys. J. E, 12, 223 (2003).

28 J. Ray and G. S. Manning, Langmuir 10, 2450 (1994).

${ }^{29}$ N.E. Jackson, B.K. Brettmann, V. Vishwanath, M. Tirrell, J.J. de Pablo, ACS Macro Letters, 6, 155-160, (2017).

30 A. Kabalnov, H. Wennerström, Langmuir 12, 276-292 (1996). 